\title{
OPTIMIZATION OF THE DCE-CT PROTOCOL USING ACTIVE IMAGING
}

\author{
Raphael Prevost ${ }^{1}$, David L. Buckley ${ }^{2}$ and Daniel C. Alexander ${ }^{3}$ \\ ${ }^{1}$ ENSTA ParisTech, Paris, France \\ ${ }^{2}$ Division of Medical Physics, University of Leeds, Leeds, UK \\ ${ }^{3}$ Centre for Medical Image Computing, Department of Computer Science, \\ University College London, London, UK
}

\begin{abstract}
This paper uses experiment design optimization to obtain the optimal placement of scans during dynamic contrast enhanced computed tomography. Here we construct and minimize an objective function based on the Cramer-Rao lower bound (CRLB) to optimize the accuracy of the estimators. Experiments based on simulated data are performed to compare our scheme with current sampling methods. Results reveal a significant reduction in error and variation of the fitted parameters while simultaneously reducing the number of scans, and thus the radiation dose received by the patient. We also show that our acquisition scheme is robust to the parameter values, easily adaptable to various parts of the body and therefore has great clinical potential.
\end{abstract}

Index Terms - computed tomography, dynamic contrast enhanced imaging, optimal sampling, Cramer Rao lower bound

\section{INTRODUCTION}

Dynamic contrast-enhanced imaging with computed tomography (DCE-CT, also known as perfusion CT) is a noninvasive in-vivo technique that provides valuable information about tissue and tumor microvascular function. The technique measures the uptake of a contrast agent injected into the patient, by fitting a tracer kinetic model to the time course of the signal. This procedure quantifies physiological parameters (e.g. blood flow, vessel permeability, blood volume) that are useful for tumor grading, prognosis and treatment monitoring $[1,2,3,4,5]$.

The signal time course comes from repeated acquisitions during an exam, typically lasting a few minutes, with some fixed temporal schedule. The quality of the parameter estimates depends on the number and the schedule of the acquisitions. However, since the patient receives a dose of ionizing radiation with every image taken, a compromise has to be found between the accuracy and precision of the parameters and the safety of the patient. The choice of imaging schedule is usually empirical. The tracer concentration increases rapidly in the first few tens of seconds after injection and subsequently decays more slowly. Accordingly, the standard schedules employed have two phases: an initial phase of acquiring successive images with as little separation as possible and a second phase with larger separation [2]. One such scheme has an initial phase of 60 acquisitions with separation $\Delta t_{\text {min }}=1 \mathrm{~s}$, limited by the rotation time of the scanner, and the second phase contains around 10 images with separation of 30s.

In this work, we use the active-imaging paradigm $[6,7]$ and a tracer kinetic model to identify the optimal imaging schedule. This both reduces the radiation dose and increases parameter estimate accuracy and precision. Preliminary work by Bisdas et al. [8] uses simulations to demonstrate that exponentially increasing spacing in the second phase improves on the standard fixed spacing. Here we provide a much more general optimization over the whole space of allowable schedules.

Section 2 introduces the model and schedule optimization algorithms. Section 3 shows the results of the optimization and tests the performance of the new schedule in Monte Carlo simulations. Section 4 provides some discussion and avenues for further work.

\section{THEORY}

\subsection{Tracer kinetics model and parameters of interest}

The contrast agent used in DCE-CT circulates from the arterial plasma to the capillaries. The artery supplies the intravascular space (compartment 1 ) at a constant flow rate $F$. The tracer then diffuses within the interstitial fluid (compartment 2 ) at a rate determined by the permeability-surface area product $P S$. The tracer kinetics can be described by the conventional two compartment exchange model (2XCM), a straightforward model that gives realistic results [4]. The model assumes that the two compartments are homogeneous and instantaneously well-mixed. The equations of the systems are

$$
\begin{aligned}
v_{1} \frac{d C_{1}(t)}{d t}= & F\left(C_{a}(t)-C_{1}(t)\right)-P S\left(C_{1}(t)-C_{2}(t)\right) \\
& v_{2} \frac{d C_{2}(t)}{d t}=P S\left(C_{1}(t)-C_{2}(t)\right),
\end{aligned}
$$


where $C_{i}$ and $v_{i}$ are respectively the tracer concentration and the volume of the $i^{\text {th }}$ compartment. Thus the impulse residue function $R$ (ie. tissue response to a unit impulse function) of the system is a biexponential function

$$
R(t)=A \exp (\alpha t)+(1-A) \exp (\beta t),
$$

where $\alpha, \beta$ and $A$ depend only on the parameters of interest : $F, P S, v_{1}, v_{2}$.

The tissue concentration $C_{t i s s}$ that we actually measure is given by

$$
C_{t i s s}(t)=F \int R(t) C_{a}(t-\tau) d \tau
$$

where

$$
C_{a}(t)=\left\{\begin{array}{cc}
\frac{C_{0}}{b^{a+1} \Gamma(a+1)} t^{a} e^{-t / b} & \text { if } t>0 \\
0 & \text { otherwise }
\end{array}\right.
$$

is a simplified version of the tracer concentration in the artery, which we obtain by fitting a gamma function to measurements from an arterial region in a clinical data set.

\subsection{Active Imaging}

The active-imaging framework uses a parametric model relating the image intensity to tracer kinetic parameters and identifies the optimal imaging parameters for estimating the physiological parameters. In short, it solves the optimal experiment design problem for quantitative imaging. The technique has proved successful in quantitative MRI for improving parameter estimates [6] and even revealing previously inaccessible parameters [7]. Here we use the approach to find the optimal CT schedule for estimating the parameters $F, P S, v_{1}$ and $v_{2}$ of the model above.

The goal is to minimize simultaneously the variances $\sigma_{i}^{2}$ of every model parameter $p_{i}$. The parameters have different scales, so we normalize them by the inverse square of the parameters values and consider the objective function $\sum_{i=1}^{n} p_{i}^{-2} \sigma_{i}^{2}$. However, the $\sigma_{i}^{2}$ are unknown so we use the Cramer-Rao lower bound (CRLB) [9] in their place. For Gaussian noise with standard deviation $\sigma$, the $i j^{t h}$ term of the Fisher information matrix $\mathbf{J}$ is :

$$
\begin{aligned}
\mathbf{J}_{i j}(\mathbf{t})=\frac{1}{\sigma^{2}} \sum_{n=1}^{N} \frac{\partial C_{t i s s}(}{} & \left.F, P S, v_{1}, v_{2} ; t_{n}\right) \\
\partial p_{i} & \\
\times & \frac{\partial C_{t i s s}\left(F, P S, v_{1}, v_{2} ; t_{n}\right)}{\partial p_{j}}
\end{aligned}
$$

where $\mathbf{t}=\left(t_{1}, t_{2}, . ., t_{N}\right)$ is the schedule of imaging times. The diagonal elements of $\mathbf{J}^{-1}$ provide the CRLBs, which are lower bounds on the variances of the corresponding parameters that often correlate well with the true variance [9]. Thus

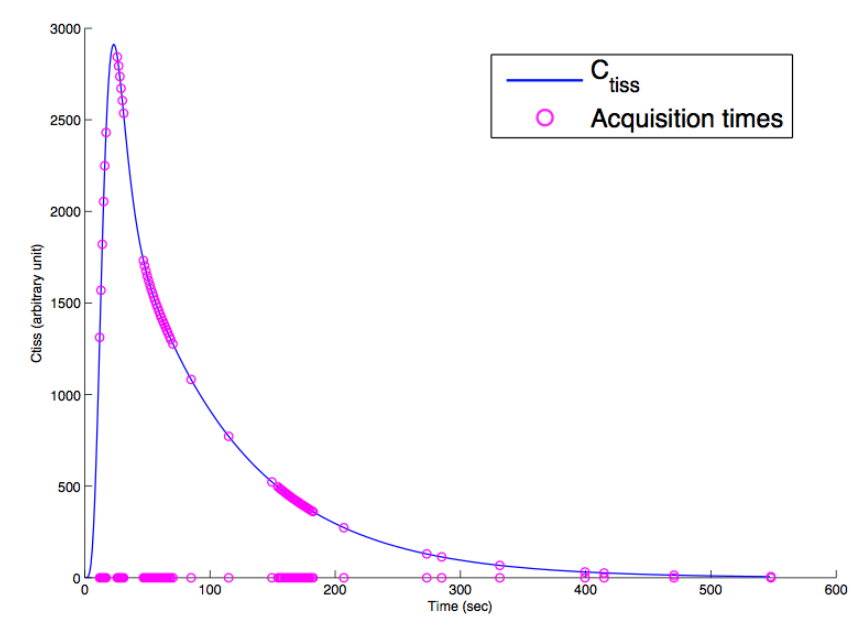

Fig. 1. Plot of the $C_{t i s s}$ curve with the a priori parameter settings used for the optimization, and the acquisition times denoted as circles.

we minimize the following objective function with respect to the schedule $t$ :

$$
V=\sum_{i=1}^{4} p_{i}^{-2}\left[\mathbf{J}^{-\mathbf{1}}\right]_{i i} .
$$

The function $V$ depends on the parameters $p_{1}, . ., p_{4}$, so the solution does too. The optimization requires a priori values for the parameters and our results are specific for this set of parameters. Unless otherwise specified, we will assume in this paper $F=70 \mathrm{ml} / \mathrm{min} / 100 \mathrm{~g}, P S=17 \mathrm{ml} / \mathrm{min} / 100 \mathrm{~g}, v_{1}=5$ $\mathrm{ml} / 100 \mathrm{~g}, v_{2}=20 \mathrm{ml} / 100 \mathrm{~g}$. These values correspond to invivo measurements from extracranial head and neck tumors [8].

Following [7], we minimize the objective function using SOMA [10] with five repeated runs to check for convergence in each of the experiments that follow. The stochastic search is essential to avoid local minima and find good solutions in this high dimensional optimization. The minimization is performed with a population size of 100,300 cycles of migrations and otherwise default settings. The algorithm has a computation time of a few hours.

\section{EXPERIMENTS AND RESULTS}

\subsection{Full optimization}

The first experiment tests the hypothesis that the activeimaging optimization can find better schedules than the standard schedules. Thus we run the optimization with the only constraint on the schedule being $t_{i+1}-t_{i} \geq \Delta t_{\text {min }}$. Figure 1 shows the optimized schedule with $N=70$.

Most consecutive acquisition times are separated by $\Delta t_{\text {min }}$. The measurements form four main clusters, which 


\begin{tabular}{|c|c|c|c|}
\hline $\begin{array}{c}\text { Standard } \\
\text { protocol }\end{array}$ & $\begin{array}{c}\text { Bisdas et al. } \\
\text { protocol }\end{array}$ & $\begin{array}{c}\text { Full } \\
\text { optimized }\end{array}$ & $\begin{array}{c}\text { Parametric } \\
\text { optimized }\end{array}$ \\
\hline 9.44 & 10.9 & 9.03 & 6.81 \\
\hline
\end{tabular}

Table 1. Values of the objective function (arbitrary unit) for the standard protocol, the schedule proposed by Bisdas et al. [8], and the solutions found in experiment 3.1 and 3.2

correspond to periods when the measurement is particularly sensitive to different parameters. The first cluster during the initial rise in the signal is sensitive to $F$; the second cluster near the peak is sensitive to $F, v_{1}$ and $P S$; the third cluster during the decreasing in the concentration is sensitive to $v_{1}$, $P S, v_{2}$; the fourth cluster is sensitive to $v_{2}$ and $P S$. A few other measurements are spaced out in between the four clusters. We consistently see the same pattern in repeated runs of the optimization. The scattered measurements appear to be important to know the general form of the curve.

\subsection{Parametric approach}

The full optimization in section 3.1 is very high dimensional so the global optimum is hard to find. However, the experiment suggests a broad form of the optimal schedule. In the second experiment, we add constraints to the schedule to give it the form of the optimal solution and reduce the dimension of the optimization. Specifically, we describe the 4 clusters of measurements with minimal separation with only 7 parameters $N_{1}, N_{2}, N_{3}, T_{1}, T_{2}, T_{3}, T_{4}$ where $N_{i}$ and $T_{i}$ denote respectively the number of acquisitions and start of each cluster. For example, the acquisition times of the first cluster are $T_{1}, T_{1}+\Delta t_{\text {min }}, T_{1}+2 \Delta t_{\text {min }}, . ., T_{1}+\left(N_{1}-1\right) \Delta t_{\text {min }} . N$ is fixed so $N_{4}$ is not a parameter.

Preliminary experiments reveal that the extra measurements between the clusters in the schedule in Figure 1 are important. We include them in addition to the parametrized schedule as 10 measurements starting after the end of cluster 3 with exponentially-increasing intervals. Figure 2 compares all the schedules. Table 1 shows that the optimized schedules have a lower $V$ than the standard protocols. The parametric optimization problem is much simpler to solve and we find a schedule with even lower $V$ in only a few tens of minutes. The next section considers only optimized parametric schedules.

\subsection{Simulations and evaluations}

The goal of these experiments is to compare the performance of schedules. In each experiment, we sample $C_{\text {tiss }}$ according to the schedule, add Gaussian noise for baseline SNR of 10, fit the model to estimate the parameters and repeat for 1024 independent trials. We compute two performance criteria:

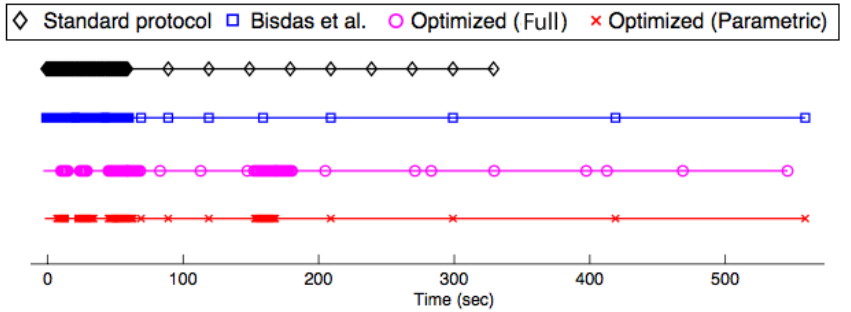

Fig. 2. Comparison between the different schedules. The protocols do not have the same duration because the last acquisition time is not fixed but optimized like the others. The parametric optimized schedule has $N_{1}=9, N_{2}=10, N_{3}=13$, $N_{4}=12, T_{1}=9 s, T_{2}=23 s, T_{3}=47 s, T_{4}=150 s$.

\begin{tabular}{|c|c|c|}
\hline $\begin{array}{c}\text { Protocol \& nb of scans } \\
\text { (\% of param. variation) }\end{array}$ & $\begin{array}{c}\text { CV } \\
\text { (Max/Mean) }\end{array}$ & $\begin{array}{c}\text { Error } \\
\text { (Max/Mean) }\end{array}$ \\
\hline Standard 70 scans (0\%) & $17.8 \%$ & $2.3 \%$ \\
Standard 70 scans (10\%) & $19.2 \% / 17.7 \%$ & $2.8 \% / 2.3 \%$ \\
Standard 70 scans (20\%) & $21.3 \% / 17.9 \%$ & $3.4 \% / 2.5 \%$ \\
\hline Bisdas 70 scans (0\%) & $14.3 \%$ & $1.8 \%$ \\
Bisdas 70 scans (10\%) & $14.7 \% / 13.8 \%$ & $2.2 \% / 1.7 \%$ \\
Bisdas 70 scans (20\%) & $15.5 \% / 14 \%$ & $2.0 \% / 1.7 \%$ \\
\hline Optimized 70 scans (0\%) & $10.0 \%$ & $0.8 \%$ \\
Optimized 70 scans (10\%) & $11.7 \% / 10.5 \%$ & $1.1 \% / 0.9 \%$ \\
Optimized 70 scans (20\%) & $12.6 \% / 10.9 \%$ & $1.6 \% / 1.0 \%$ \\
\hline Optimized 55 scans (0\%) & $10.6 \%$ & $0.8 \%$ \\
Optimized 55 scans (10\%) & $12.4 \% / 11.6 \%$ & $1.0 \% / 1.0 \%$ \\
Optimized 55 scans (20\%) & $13.9 \% / 12.0 \%$ & $1.6 \% / 1.1 \%$ \\
\hline Optimized 35 scans (0\%) & $14.1 \%$ & $1.3 \%$ \\
Optimized 35 scans (10\%) & $17.1 \% / 15.1 \%$ & $1.4 \% / 1.1 \%$ \\
Optimized 35 scans (20\%) & $17.3 \% / 14.9 \%$ & $2.0 \% / 1.4 \%$ \\
\hline
\end{tabular}

Table 2. Values of the mean error and coefficient of variation over the four parameters of interest, for different protocols, with fixed parameters values $(0 \%)$ or varying in different ranges $(+/-10 \%$ or $20 \%)$

the relative error $E_{i}=\left|\bar{p}_{i}-p_{i}\right| / p_{i}$ (where $\bar{p}_{i}$ is the mean of the 1024 parameter estimates) and the coefficient of variation $C V_{i}=S T D_{i} / p_{i}$, where $S T D_{i}$ is the standard deviation of the 1024 estimates of $p_{i}$.

We run three experiments with differents values of the parameters. First, we simulate $C_{\text {tiss }}$ curves with the exact values of the parameters used to optimize the schedule. The second (resp. third) experiments are designed to test whether the performance of our solution is sensitive to the value of the parameters. Thus we take random values for the parameters within a range of $+/-10 \%$ (resp. 20\%) of the values with which the schedule has been optimized. We simulate $C_{\text {tiss }}$ curves with these sets of parameters and compute the worst-case and average of the criteria. All the results are summarized in Table 2. 
The optimized schedule with 70 measurements provides parameters estimates with much lower CVs and errors than the standard schedules. The results reveal that our schedule is robust to variations of parameters within a range of $10 \%$ or $20 \%$. If we are particularly interested in minimizing the radiation dose received by the patient, it is possible to reduce the number of measurements. With 55 acquisitions, the estimates still have a lower $\mathrm{CV}$ and error than from other schedules with 70 measurements. Even with only 35 measurements, the error is reduced although the estimates are less stable and the CVs are a bit higher. This suggests that we can reduce the radiation dose by half and still have a low error, which has important clinical implications.

\section{DISCUSSION}

The experiments show that optimizing the acquisition schedule in DCE-CT offers significant opportunity to both reduce radiation dose and improve parameter estimates.

We repeated our Monte-Carlo simulations with different SNR. Results show that our optimized schedules provide a lower error and $\mathrm{CV}$ than the standard protocols whatever the SNR, but the difference is more significant for lower SNR.

The results shown in this paper were computed with parameter values that reflect extracranial tumors. Similar experiments with values typical of rectal tumors and normal tissues, and cerebral meningioma also provide improved parameter accuracy and precision using a similar parametric model (composed of 4 clusters of measurements and several individual measurements) although $N_{1}, N_{2}, N_{3}, T_{1}, T_{2}, T_{3}$ and $T_{4}$ depend on the specific parameters values chosen.

In this study, we used active imaging in static mode, which means that we computed the optimal schedule before the actual examination, but we could use the same principles dynamically. If we estimate the parameters during the exam, we could optimize the remaining acquisition times and thus tailor the protocol to the patient. However, preliminary experiments suggest only minor improvements.

The way the contrast product is injected into the patient can also be optimized. Results of preliminary experiments revealed that the best way is to inject the contrast product as a bolus (i.e. all at once at the start of the exam), which corresponds to the standard protocol.

Limitations of our work remain. We only used simulated data. Future work will employ real clinical data to confirm that our solution provides better estimates and can be used in practice. Moreover, in this study we used an independently measured arterial input function. That is, it was not measured using the same schedule as the tissue tracer concentration. Further work will include a more realistic model of the arterial input (e.g. [11]) and the optimization of the schedule for measuring both the tissue and arterial tracer concentration. It will also include measured arterial input functions and explore the influence of their variability.

\section{REFERENCES}

[1] W.T. Sobol and J.K. Cure, "Can in vivo assessment of tissue hemodynamics with DCE-CT be used in the diagnosis of tumors and monitoring of cancer therapy outcomes," Radiology, vol. 232, pp. 631-632, 2004.

[2] M.A. Haider, M. Milosevic, A. Fyles, and T.P.L. Roberts, "Assessment of the tumor microenvironment in cervix cancer using dynamic contrast enhanced CT, interstitial fluid pressure and oxygen measurements," Int $J$ Radiat Oncol Biol Phys, vol. 62, no. 4, pp. 1100-1107, 2005.

[3] D.V. Sahani, S.P. Kalva, L.M. Hamberg, and T.Y. Lee, "Assessing tumor perfusion and treatment response in rectal cancer with multisection CT: initial observations," Radiology, vol. 234, no. 3, pp. 785-792, 2005.

[4] D. Cheong, T. Lim, and T.S. Koh, "Dynamix contrastenhanced CT of intracranial meningioma: Comparison of distributed and compartmental tracer kinetic models initial results," Radiology, vol. 232, pp. 921-930, 2004.

[5] G. Brix, F. Kiessling, R. Lucht, S. Darai, K. Wasser, S. Delorme, and J. Griebel, "Microcirculation and microvasculature in breast tumors: Pharmacokinetic analysis of dynamic MR image series," Magnetic Resonance in Medicine, vol. 52, no. 2, pp. 420-429, 2004.

[6] M. Cercignani and D.C. Alexander, "Optimal acquisition schemes for in vivo quantitative magnetization transfer MRI,' Magnetic Resonance in Medicine, vol. 56, no. 4, pp. 803-810, 2006.

[7] D.C. Alexander, "A general framework for experiment design in diffusion MRI and its application in measuring direct tissue-microstructure features," Magnetic Resonance in Medicine, vol. 60, pp. 439-448, 2008.

[8] S. Bisdas, C. Z. Foo, C. H. Thng, T.J. Vogl, and T.S. Koh, "Optimization of Perfusion CT for Imaging of extracranial head and neck tumors," Journal of Digital Imaging, vol. 22, no. 5, pp. 437-448, 2008.

[9] S.M. Kay, Fundamentals of statistical signal processing: estimation theory, Prentice Hall, 1993.

[10] G.C. Onwubolu and B.V. Babu, New Optimization Techniques in Engineering, Springer, 2004.

[11] M.A. Horsfield, J.S. Thornton, A. Gill, H. R. Jager, A.N. Priest, and B. Morgan, "A functional form for injected MRI gd-chelate contrast agent concentration incorporating recirculation, extravasation and excretion," Physics in Medicine and Biology, vol. 54, no. 9, pp. 2933-2949, 2009. 